\title{
Investigation of an automated dry fiber preforming process for an aircraft fuselage demonstrator using collaborating robots
}

\author{
Mona Eckardt ${ }^{1} \cdot$ Andreas Buchheim $^{1} \cdot$ Tobias Gerngross $^{1}$
}

Received: 4 May 2015/Revised: 12 May 2016/Accepted: 13 June 2016/Published online: 23 June 2016

(C) Deutsches Zentrum für Luft- und Raumfahrt e.V. 2016

\begin{abstract}
High-performance carbon fiber-reinforced plastics (CFRP) see a continuous growth of their share in structural weight. In particular, in Aerospace, this includes the production of large components, where the key issue of significant high costs remains persistent. The Center for Lightweight Production Technologies in Augsburg as a part of the German Aerospace Center (DLR) addresses this problem with solutions for process automation to increase repeatability, process robustness, and cost-efficiency. This paper presents an approach for automated preforming of large CFRP parts by means of collaborative robots. Investigations include process development for robotic handling of large-carbon fiber cut- pieces and validation on a full-scale demonstrator. The experimental verification is focused on a demonstrator geometry that represents a fuselage section of a typical short- to mid-range aircraft. The work is completed by a discussion about the challenges, solution approaches, and observations. The presented semi-automated preforming process with collaborating robots is an important intermediate step on the way to a fully automated production process for large Aerospace parts made of CFRP.
\end{abstract}

Keywords Aircraft - Automated production - CFRP . Gripper · Non-crimp fabric $\cdot$ Preforming

This paper is based on a presentation at the German Aerospace Congress, September 16-18, 2014, Augsburg, Germany.

Mona Eckardt

mona.eckardt@dlr.de

1 Center for Lightweight Production Technology, Institute of Structures and Design, German Aerospace Center, Am Technologiezentrum 4, 86159 Augsburg, Germany

\section{Introduction}

An important factor for the aviation industry is cost efficiency, which can be translated into requirements for weight reduction of aircrafts with a decrease of fuel consumption. One approach to reduce the aircrafts weight is the use of composite materials for structural parts. Beginning in the 70s, Airbus used for its first aircraft (Airbus A300) CFRP for structural components. At that time, the proportion was only $5 \%$ of the total mass of the aircraft [1]. In 2015, the new Airbus A350 XWB is produced with a proportion of CFRP of about $52 \%$ in structural weight. Although CFRP sees this continuous growth of their share in structural weight, the key issue of significant high costs remains persistent.

In particular, in aerospace, the manufacturing of large CFRP parts still involves many manual process steps, leading to reduced reproducibility and the necessity of rework.

The Center for Lightweight Production Technologies in Augsburg addresses this problem with solutions for process automation. This work addresses large CFRP parts with a variety of target structures including single and double curvatures that are made of dry carbon fiber textiles using a vacuum-assisted process for infiltration [2-4]. Together with the DLR Stuttgart, a project was launched to develop tools and technologies towards an automated process chain. As demonstrator geometry with single curvature, half a fuselage section including stringers has been chosen. The project is used to realize a step-by-step development of an automated production process. Progress is being validated in a series of half shell demonstrators starting from a manual lay-up, followed by semi-automation and resulting in a future, fully automated approach. 


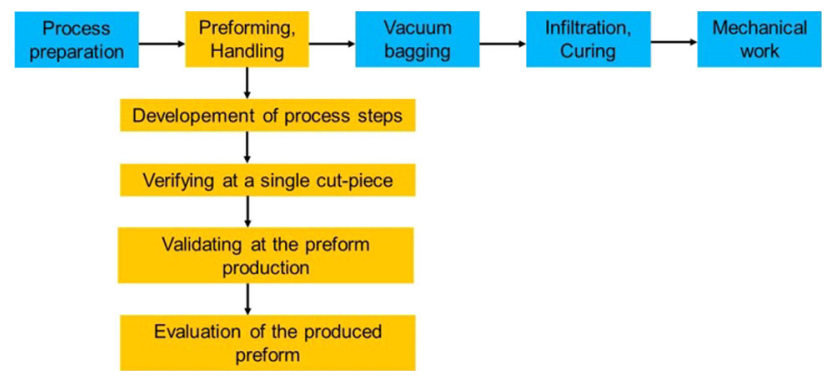

Fig. 1 Process chain for CFRP production [5]

The main focus in this paper is the development of an automated preforming process, where the handling of dry fiber fabrics with given grippers and collaborating robots is examined. Investigations include the pick-up, transport. and drop-off (draping) process of the carbon fiber fabrics from a table to the correct position on a half shell tooling. These preforming process steps are initially developed and verified by tests with a single cut-piece of the plybook. Next, they are validated together with scientific findings of the digital process chain [4] by an application for the semiautomated production of the half shell demonstrator. A basis for this work is the manual preforming process which is used to derive the automated preforming steps. Figure 1 shows the whole process of production for CFRP components. The focus in this paper is highlighted in yellow.

\section{The target structure}

The target structure is a cylindrical half shell with local reinforcements for a frame and six stringers which is comparable to a single-aisle fuselage geometry. This demonstrator has a radius of $1977 \mathrm{~mm}$ with a depth of $2000 \mathrm{~mm}$ and a thickness between 2.4 and $4.8 \mathrm{~mm}$. Figure 2 shows the demonstrator which has been built by hand

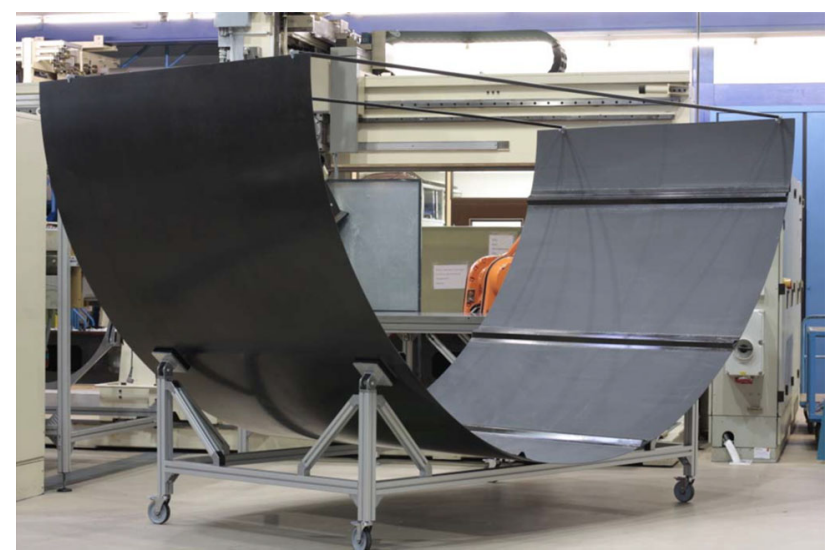

Fig. 2 Half shell demonstrator

to define the scope for automation and identify key challenges.

The fuselage segment has been chosen as a representative demonstrator to get more knowledge along the complete process chain of an automated production process of large aircraft structures. The properties of the demonstrator regarding dimension, curvature, and accuracy requirements were deemed to be representative for other aerospace components of CFRP (e.g., wing, pressure bulkhead, and cargo door). Therefore, a knowledge transfer is possible.

A new approach in this project is the use of dry carbon fiber fabrics in combination with thermoplastic stringers [Carbon Fiber (CF)/Polyetherimid (PEI)] which are bonded during a vacuum-assisted resin infusion (VARI). Relating to the preforming process, this approach provides the chance to get experiences of handling, draping, and fixation of large dry fiber cut-pieces by grippers in combination with collaborating robots on a female tooling. In addition, the automated integration of sub-preforms and stringers can be tested.

For a realistic test setup based on existing industrial components, different features are integrated in the demonstrator. A multidirectional structure of cut-pieces that vary in size and shape; local reinforcements with different ramp gradients $(1: 10,1: 20)$ in circumferential and longitudinal directions and end-to-end cut-piece application are used. The materials for the demonstrator are biaxial carbon fiber (Tenax HTS40 F13 $12 \mathrm{~K}$ ) non-crimp fabrics manufactured by Saertex $\mathrm{GmbH}$. The mass per-unit area is $268 \mathrm{~g} / \mathrm{m}^{2}\left(0 / 90^{\circ}\right)$ and $266 \mathrm{~g} / \mathrm{m}^{2}\left( \pm 45^{\circ}\right)$ for the different fiber orientations. The mass includes $6 \mathrm{~g} / \mathrm{m}^{2}$ of binder (Momentive Epikote Resin 05311) on one side of the fabrics which is used to fix the cut-pieces among each other after they are placed at the tooling. A schematic construction of a $0 / 90^{\circ}$-fabric is shown in Fig. 3. The $0^{\circ}$ fibers are placed at the $90^{\circ}$ fibers, and for fixation, they are sewed.

These kinds of non-crimp fabrics (NCF) were selected, because they offer the best compromise regarding to handling, draping, and the mechanical properties of the cured laminate. The reduced drapability is still sufficient for the single-curved shape of the demonstrator. An advantage is that a low number of material defects appear in large cutpieces during material handling. In addition, an increase of

Fig. $30 / 90^{\circ}$ schematic fabric construction

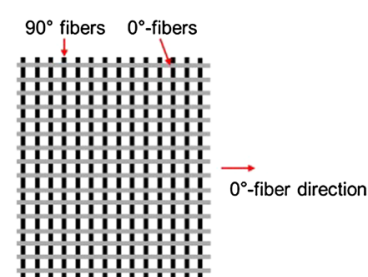


the lay-up rate is possible using multiaxial fabrics or NCF with higher masses per-unit area. Due to reduced fiber undulation in NCFs compared to weaves, improved mechanical properties are achieved.

\section{The plybook}

The flat shell surface of the demonstrator is generated by rectangular cut-pieces which are called skin layers. The size of these layers is $1989 \mathrm{~mm}$ in the length and $1034 \mathrm{~mm}$ of width. 56 skin layers are used to build the shell surface. Figure 4 shows the order of a sequence of skin layers, where six of them are used to fill the $6204 \mathrm{~mm}$ circumferential length of the tooling. Cut-pieces 1 and 7 are cut from one large cut-piece. In total, eight sequences of skin layers are applied.

Additional layers for local reinforcement are placed in longitudinal and circumferential direction. Frame layers in circumferential direction are used in the same number and order as skin layers. The sequences of frame layers are inserted in alternating sequence with the skin layers to build a symmetric structure. The size of the layers varies in longitudinal direction between 706 and $784 \mathrm{~mm}$ to create a stair structure which is used to place the local reinforcements in circumferential direction. Figure 5 shows the global position of the frame layers.

Twelve stringer bases used as thickenings are distributed at the circumference. The stacking of each stringer base contains of eight cut-pieces in a symmetric order. The single layers of each stringer base are fixed among each other before they are placed on the half shell tooling. The size of the stringer bases is designed to fit at one end with

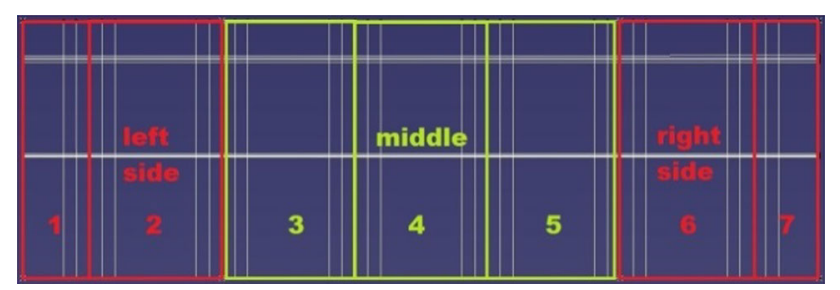

Fig. 4 Order of the cut-pieces in top view

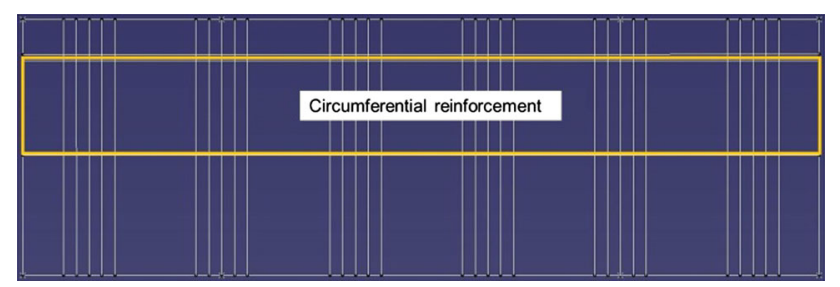

Fig. 5 Position of the frame layers in top view

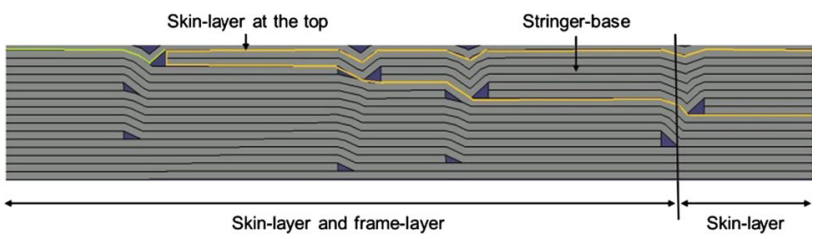

Fig. 6 Structure of the layers

the frame layers and at the opposite end with the manufacturing edge of part (MEOP). For this reason, there are six short stringer bases with a length between 268 and $318 \mathrm{~mm}$ and six long stringer bases with a length between 933 and $958 \mathrm{~mm}$. The width of both versions varies between 139 and $184 \mathrm{~mm}$ to generate the stair structure in longitudinal direction, too. This stair structure is necessary to avoid the generation of a point of failure when the infusion is finished. Figure 6 represents schematically the structure and interaction of the different layers.

At the top of the stringer bases, one closing sequence of skin layers is applied. The reinforcements in longitudinal and circumferential direction are designed to generate a flat surface for six omega stringers made of cured thermoplastic CFRP. The stringers are fixed to the stringer bases and frame layers by a melted thermoplastic fleece. The stringers are combined with a foam core to avoid cavities that are filled with resin during the infiltration. Figure 7 shows the cross section of a stringer with foam core.

In general, the sequences of skin layers and frame layers are built of cut-pieces with a fiber orientation of $\pm 45^{\circ}$ or $0 / 90^{\circ}$. Some sequences are built of cut-pieces with fiber orientations of $\pm 45^{\circ}$ at the edges in circumferential direction and of cut-pieces with a fiber orientation of $0 / 90^{\circ}$ in the middle. These ones mostly absorb shear stress and torsion $\left( \pm 45^{\circ}\right)$ and compression and tension $\left(0 / 90^{\circ}\right)$.

The plybook was generated with the CAD program CATIA Composite Part Design (CPD). This is where stacking, maximum shear angle of the material during draping, seed point as fixation, and start position for draping and propagation type are defined. The applied stacking is described at the beginning of this chapter. The basis for this stacking is the real surface of the tooling which was scanned by a laser-tracking system and rebuild in CATIA. A warning

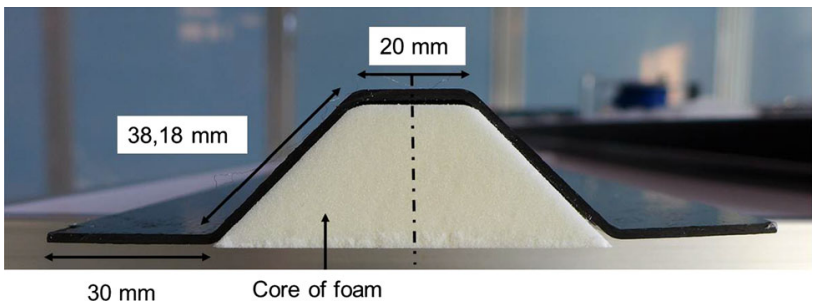

Fig. 7 Stringer with a foam core 
shear angle of $15^{\circ}$ and a limit shear angle of $30^{\circ}$ are defined for draping. The seed point is defined as the center of gravity for each cut-piece, and for the propagation type, the standard one of CATIA CPD is chosen. Due to the simple geometry of the tooling, it is not necessary to shear the cut-pieces during draping. It follows that the warning shear angle will never appear (manual and automated process), and the standard propagation type of CATIA is appropriate for this plybook. The influence of the seed point as a material fixation during draping is described below.

\section{The basis for automated preforming}

\subsection{Material handling derived from manual process}

The automation of the preforming process can reduce the process time and facilitate a repeatable quality of the product. The general procedure of the automated preforming process is comparable to the manual one, where between two and four workers pick up, transport, position, and drape the cut-pieces like a tablecloth from a table onto a tooling. Grippers which are used as the handling system during the automated process are built of individual modules to be adjusted to the target geometry. With these grippers, the cutpieces can be handled in the same way, as it would be done during the manual process. Figure 8 shows an illustration of manual and automated material handling.

For both processes, the material is held in the shape of the catenary that is formed due to the cut-piece's own weight. The automated transport of the cut-piece from one location to another is done by collaborating robots which perform geometrically linked movements. Again, the manual and automated movement to the drop-off position is comparable. The main difference between the manual and automated process is the material draping (see Sect. 4.1).

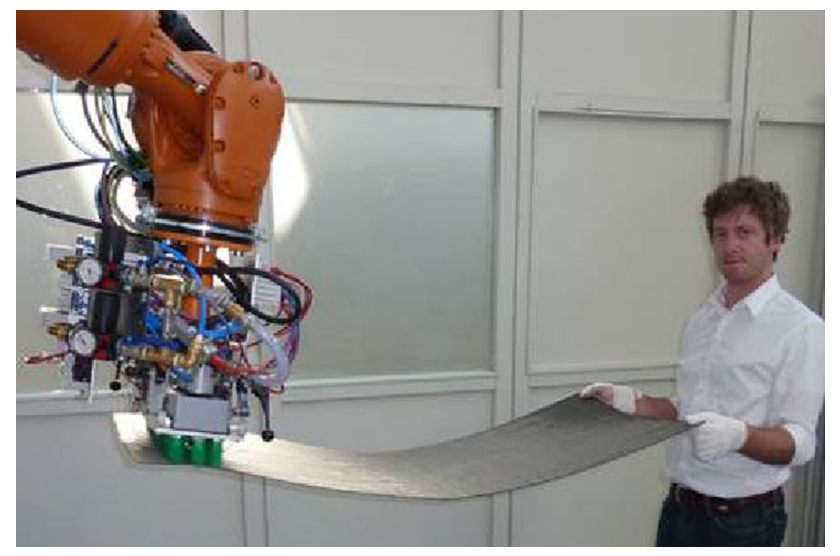

Fig. 8 Manual and automated handling of a large-carbon fiber cutpiece [6]
In general, the aims of the manual and automated process are the same ones. In detail, they are:

1. Cut-piece handling by the grippers/hands without fiber undulation and deformation of the fiber orientation.

2. Draping of the cut-pieces without distortion, wrinkles, and bridging.

3. Precise positioning of the cut-pieces (minimal difference to the given position in CAD).

\subsection{Draping of dry fiber fabrics}

The way of draping influences the quality of the product. During draping, it is important to avoid wrinkles, bridging, or a deformation of the fiber orientation to maintain the mechanical properties of the dry fiber fabrics. There is a range of possibilities to drape a cut-piece on a tooling surface. A key factor for the choice is the fixation strategy for the cut-piece and the fine-positioning of the non-fixed areas of the cut-piece on the tooling.

The part of the material which is placed first at the tooling defines the point, where the draping process starts. It is common to fix the cut-piece at one or more points (seed points) or lines (seed curves). For example, a point fixation is often used for manual processes. From this fixed point, the other areas of the cut-piece are smoothed by hand in all directions until the cut-piece is lying flat without wrinkles, folds, or bridging. For example, cutpieces with a round shape are often draped starting from a seed point, too. During this process, the non-fixed areas are arbitrarily draped in all directions which result in a not reproducible draping process. In consequence, fiber deformations can be often found. Figure 9a shows the possible draping directions from a seed point at an upper position at the cut-piece.

A line fixation of the cut-pieces is more appropriate for automated applications. It enforces a constant and uniform draping of the cut-pieces (Fig. 9b) from the fixed line. This results in a reproducible draping process with a low risk of fiber deformations. A variation of this way of draping is a process with two fixed lines (Fig. 9c) which is used for large cut-pieces. The line fixation is at both edges of the cut-piece and draping starts from these lines in the direction of each other. The chance of wrinkles, folds, or bridging increases, because any fine adjustments need to be made in advance, i.e., during the material pick-up and drop-off. The tests in Sect. 5 are discussing this difficulty.
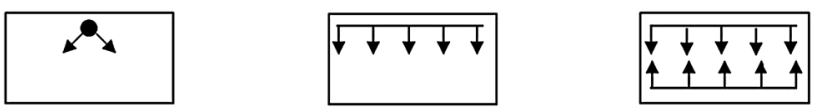

Fig. 9 Point fixation (a), line fixation (b), double-line fixation (c) 


\subsection{The catenary}

During the handling of a cut-piece by a multi-gripper system (see Fig. 8), the freely hanging part shows a deformation depending on the number of grippers, their positions, and orientations. For example, Newell and Khodabandehloo [7] developed a general shape prediction model for multi-arm robots. However, if a cut-piece would be held along two opposing edges by two parallel grippers, the freely hanging part of the cut-piece would form a natural shape similar to a catenary.

The catenary describes a rope with zero stiffness that is fixed at both sides and loaded just by its own weight. The mathematic formula is listed, which describes the catenary, in the following:

$z(x)=a \cosh \left(\frac{x-x_{0}}{a}\right)+z_{0}$.

$l=2 a \sinh \left(\frac{w}{2 a}\right)$

The variables are defined as follows (Fig. 10):

- $l=$ length of the cut-piece.

- $a=$ radius at the deepest point of the catenary.

- $w=$ distance between the fixation.

- $x / z=$ coordinates of the curve.

- $\alpha=$ inclination of the grippers/catenary.

With the length of the rope and the distance between the two fixations known, every point of the catenary can be calculated. If the grippers are to be oriented tangentially to the resulting catenary, the above equation can be used to derive the angle of inclination. Figure 11 shows a cut-piece in the shape of the catenary held by two grippers and their angle of inclination alpha [8].

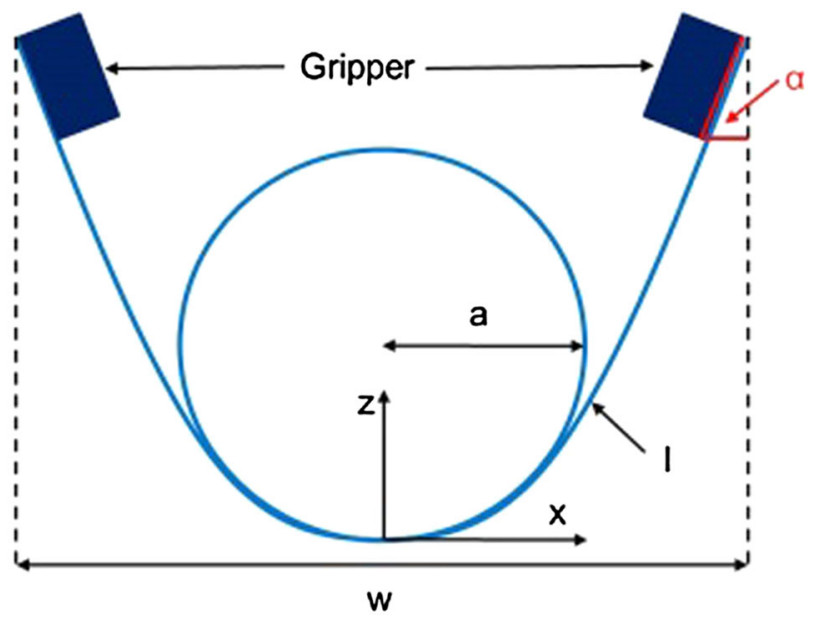

Fig. 10 Schematic view of the catenary with two grippers

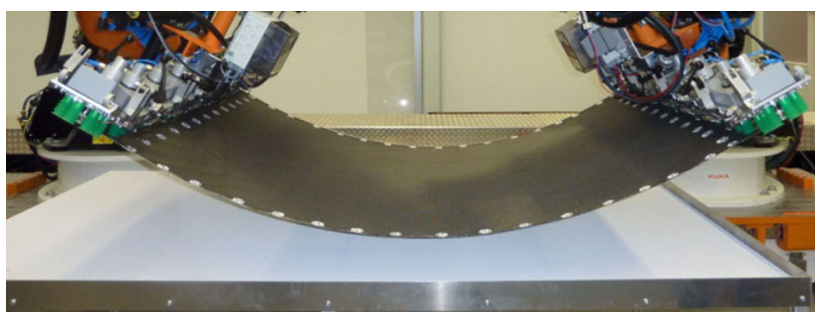

Fig. 11 Catenary curve with a cut-piece and collaborating robots

\subsection{The handling system: gripper}

For the automated handling of the dry fiber fabrics, a gripper was developed which can pick up, transport, and position large cut-pieces (Fig. 12).

The gripper is made of nine modules which are connected by joints. The module in the middle is fixed to the robot flange. The other eight ones can be rotated in $x, y$, and $z$ directions to adjust for individual surface geometries that may include single or double curvatures. To handle the cutpieces of the half shell demonstrator, the gripper is used with the orientation of $0^{\circ}$ in $x, y$, and $z$ direction. This means that the movable modules have the same orientation as the rigid one in the center. In this position, the gripper has a total length of $2000 \mathrm{~mm}$. The width of each module is about $210 \mathrm{~mm}$ and the height $300 \mathrm{~mm}$.

At the bottom of the modules, eight round bellow suction pads with a diameter of $31 \mathrm{~mm}$ and one with a diameter of $33 \mathrm{~mm}$ are mounted to handle the fabrics by vacuum suction. In different tests, these suction pads are proved to be an appropriate surface to handle dry carbon fiber fabrics $[10,11]$. A proportional valve to regulate the vacuum is used to handle fabrics with different masses perunit areas. The smaller bellows are very flexible with a low stiffness to be compressed during the placement of the cutpieces at the tooling. The bigger bellow has a higher

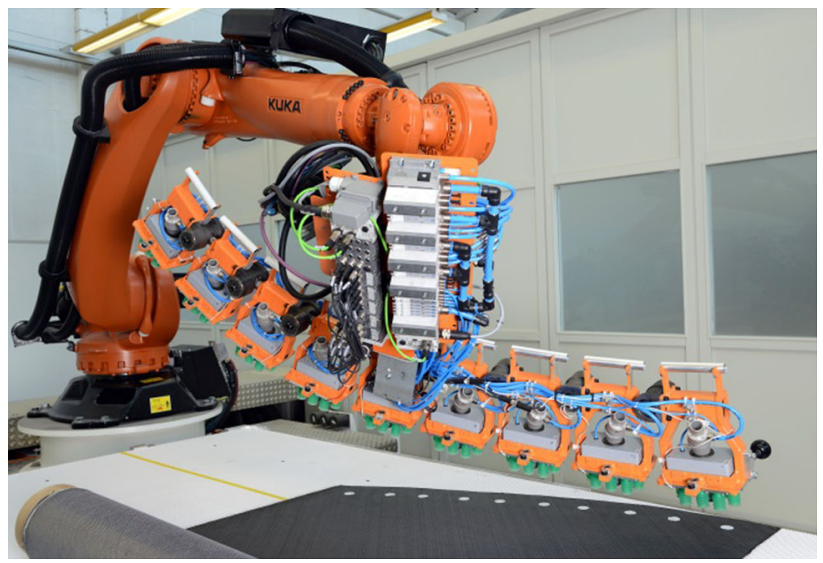

Fig. 12 Robot with a gripper for material handling [9] 
stiffness and provides more lateral stability to avoid a lateral shift of the material during pick up. Furthermore, a heating element is placed between the bellows to melt the binder and fix the material in its position. Figure 13 shows the components at the bottom of a single module.

\subsection{The positioning system: collaborating robots}

Two robots are necessary to handle the large skin layers. A special function in the robotic system called Roboteam enables a geometrical link between the robots and a work together in cooperating movements. To avoid a collision, one robot is declared as Master and the other one as Slave. The Slave is linked to the coordinate system of the Master and follows its movements. The choice which robot works as Master or Slave depends on the application.

For the preforming process of the half shell tooling, two different commands called GEOLINK and PROGSYNC are used. GEOLINK allows geometrical-linked movements which are identical of Master and Slave. Alternatively, with PROGSYNC, implemented movements start at the same time. It is not necessary for both robots to do geometric identical movements. Just the beginning of the subsequent movement is synchronized [13].

\section{Experimentical verification of an automated pick-up and draping process}

\subsection{Test setup}

The test setup is built in one of the test benches at the DLR Augsburg. It consists of two industrial robots on a linear

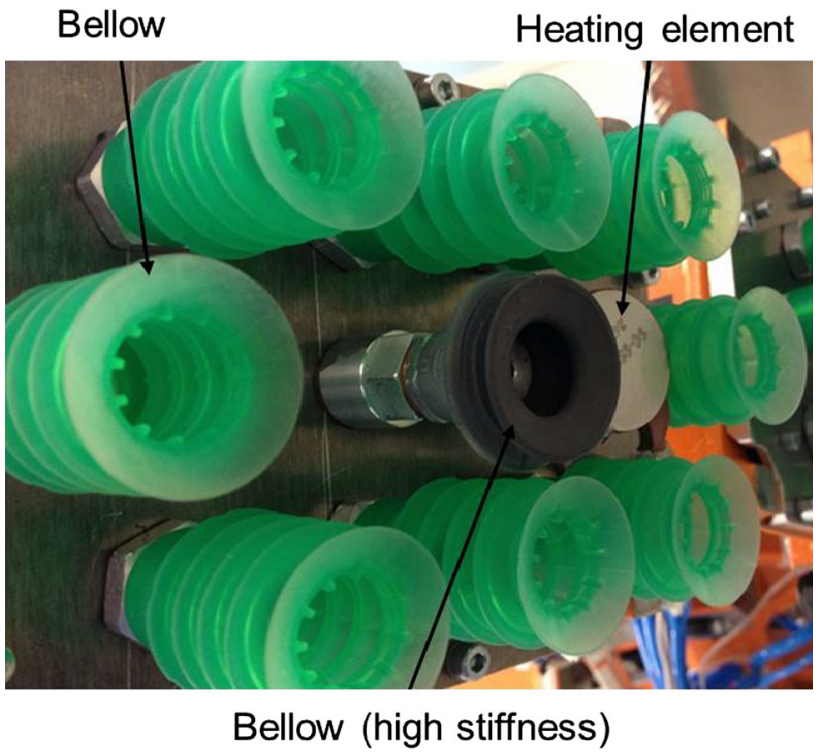

Fig. 13 Bottom side of a modul [12] axis and grippers mounted on each robot. The tooling geometry for the tests represents a fuselage section, where one of the skin layers is to be positioned, draped, and attached. A table in front of the tooling is used to provide the cut-pieces for pick-up by the grippers. The cut-pieces for the tests are of the same size as the skin layers with a fiber orientation of $\pm 45^{\circ}$. This fiber orientation has been chosen as a challenging test case, because it is more susceptible to deformation and undulation as the $0 / 90^{\circ}$ material. The complete test setup is shown in Fig. 14.

\subsection{Pick-up process}

In general, the pick-up process starts with a cut-piece lying flat at a table and ends when the cut-piece no longer touches the table and is held by the two grippers. The key requirements for the pick-up process are the prevention of any undulations or deformations of the textile (see aims of automation point 1). Towards the end of this process step, the freely hanging material will take the shape of a catenary between the two grippers (Fig. 11). To support careful handling of cut-pieces without shearing or unnecessary bending of the textile, the orientation of the grippers is to be tangentially adjusted. This means that the angles of gripper inclination are defined by the angles at both the ends of the catenary. Based on Sect. 4.3, the angles have been calculated for the handling of a skin layer by the grippers. For the following test, the inclination will be $13^{\circ}$.

The pick-up movement starts with a motion of both robots from a defined home position to a preposition which is about $30 \mathrm{~mm}$ above the cut-piece provided on the table. The home position of both robots is different, and therefore, their movement to the preposition is done independently. After reaching the position, the grippers are orientated parallel to each other and parallel to the long side of the material. Then, the grippers move to the pick-up position at the surface of the cut-piece. Preposition and pick-up position are both taught manually. The previous

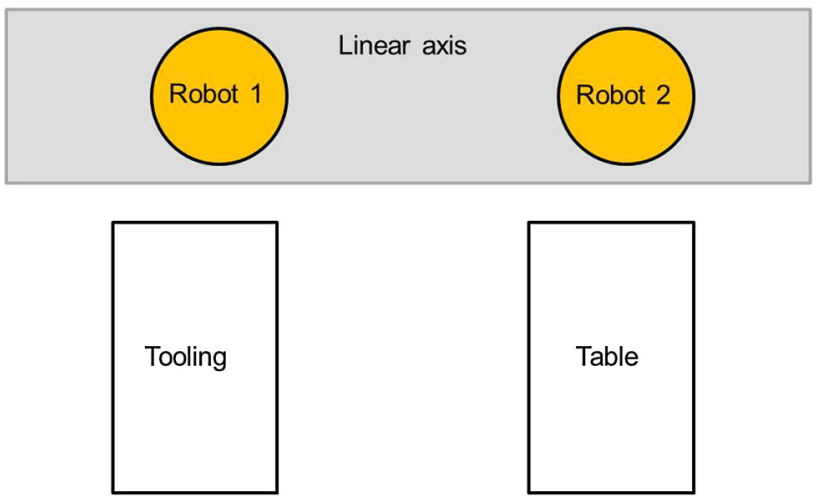

Fig. 14 Schematic test setup 


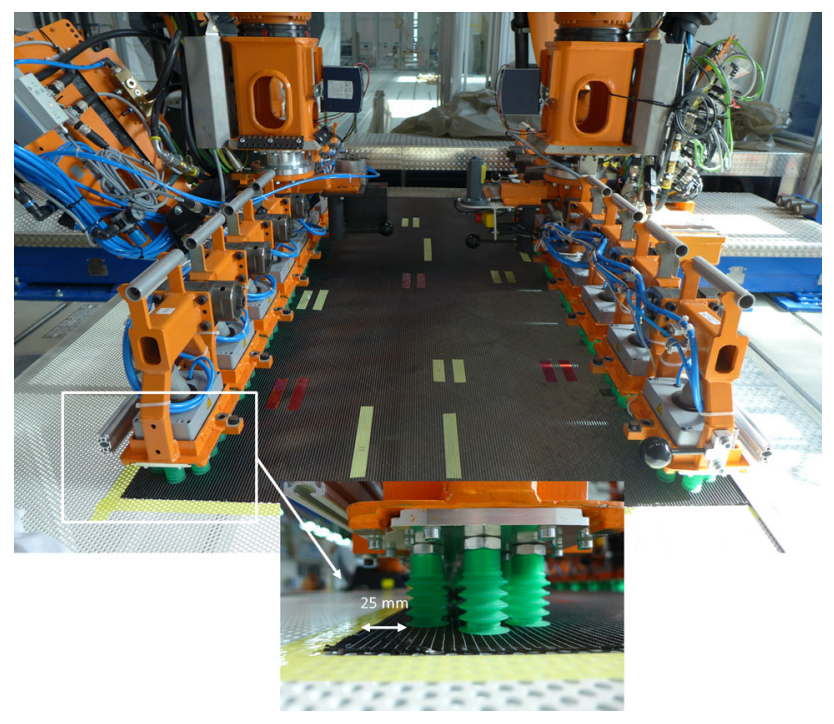

Fig. 15 Pick-up position of the grippers

tests showed that bending material near the grippers reduces grip and increases the risk of losing cut-pieces during the process. In particular, the distance between the outer edge of the grippers and the free end of the cut-piece must not be too large due to bending down from gravity. With a free edge of $25 \mathrm{~mm}$, the material was found to have enough own stability to prevent this phenomenon. Figure 15 shows the pick-up position just before activating vacuum suction of the grippers.

From the position at the material surface, Roboteam is used with the PROGSYNC function. Both grippers move at the same time $200 \mathrm{~mm}$ upwards and rotate in the opposite direction about $6.5^{\circ}$ around their longitudinal axis. The same movement is done a second time. During the short interruption between both movements, the bellows can stabilize and the chance is lower that the material is lost from the grippers. Now, the final position of the pick-up process with a distance of $400 \mathrm{~mm}$ to the table surface is reached. The angle of gripper inclination corresponds to the catenary with $13^{\circ}$. This orientation of the robots relative to each other is kept for the beginning of the drop-off process, since the distance between the grippers does not change during the transport to the half shell tooling. PROGSYNC is being used to perform this movement of the robots on the linear axis.

\subsection{Drop-off process}

For the drop-off process, it is important to choose the rightdraping strategy, regarding draping sequence and gripper movements. For the tests described below, the master is defined as the robot which will place the cut-piece at the upper position at the tooling.

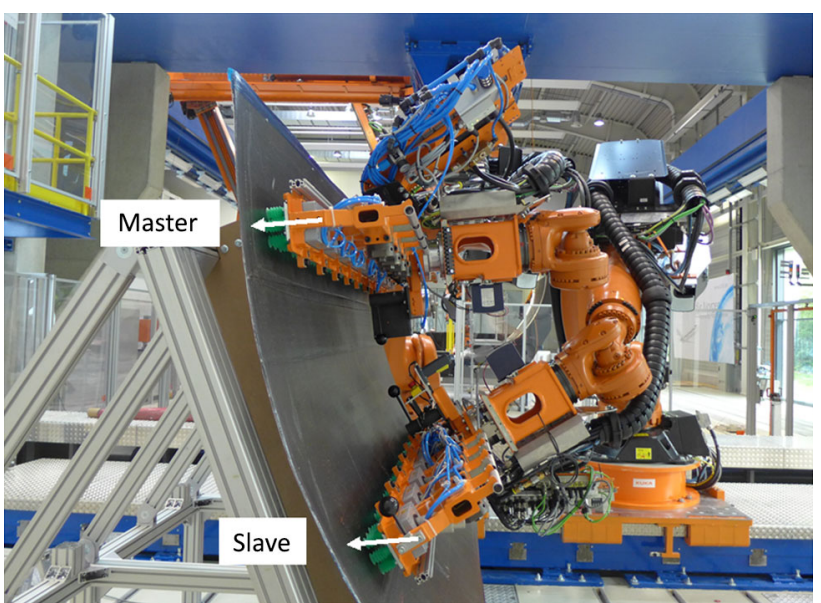

Fig. 16 Preposition with moving directions of the robots

The first strategy is a material drop-off with the GEOLINK function. When the position above the half shell tooling is reached, GEOLINK is used to perform a rotation around the longitudinal axis of the master to arrive at a preposition which is close to the surface of the half shell tooling (Fig. 16). From the preposition, GEOLINK is used again to transport the cut-piece at the drop-off position. Drop-off position and preposition of the master are taught manually and are equal for all the described drop-off tests. For the slave, it is not necessary to teach it. Its positions are dependent of the master positions due to the geometrically linkage during the transport and orientation process to the preposition.

It is defined that the master moves in perpendicular direction at the surface of the tooling. Since the slave follows the movement of the master exactly, he cannot move in perpendicular direction at the surface of the tooling. Figure 16 shows the movements of master and slave starting at the preposition.

I was observed that during its movement, the slave pushes the material and generates a wrinkle at the material when the drop-off position is reached. Figure 17 shows this undesired result.

Two reasons for this observation have been identified. First, the gripper distance spans less tooling length $\left(l_{t}\right)$ than provided by the length $\left(l_{\mathrm{f}}\right)$ of the cut-piece. Second, any gripper movement relative to the tooling surface will result in either pulling or pushing cut-pieces that already have contact to the surface. Since the GEOLINK function keeps both grippers at constant distance and orientation to each other, a perpendicular movement of the master towards the tooling surface results in a side movement of the slave. This side movement pushes the material to build a fold seen in Fig. 17. Both issues are illustrated in Fig. 18.

After this test, the cut-piece is manually placed at the table again. Visual assessment and measurement of cut- 


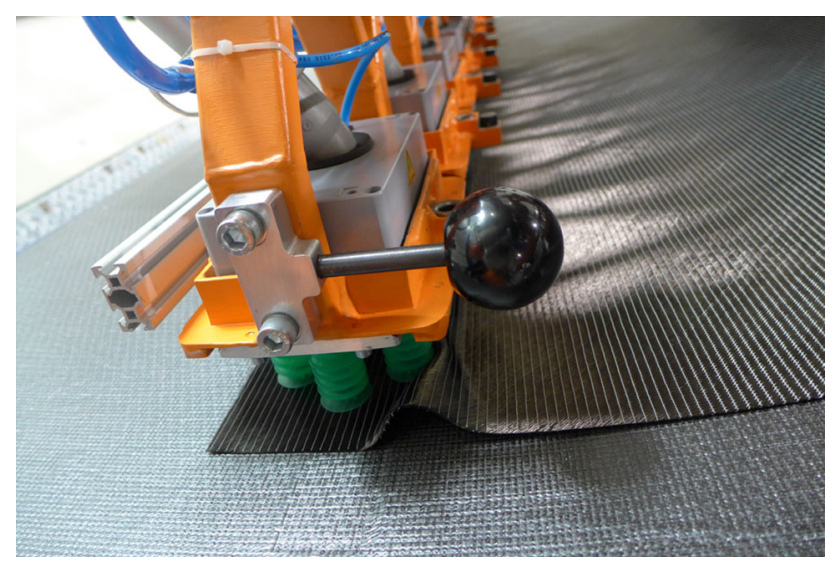

Fig. 17 Generated fold after the drop-off process

Fig. 18 Draping test 1

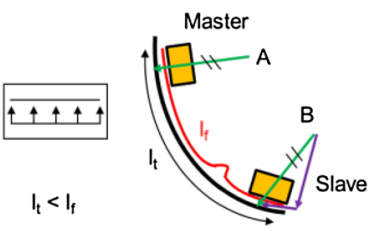

piece boundaries showed no undulation or change of fiber orientation.

In the second strategy, the focus is on elimination of the wrinkle during the drop-off process. Gripper movements are performed without a geometric linkage of the robots. Once the grippers have reached their prepositions, GEOLINK is turned off. From this position, first, the master moves perpendicular to the tooling surface, and after reaching the drop-off position, the slave starts moving, according to its local preposition, perpendicular at the tooling, too. Without GEOLINK, the robots are moving independently and in their own local coordinate systems. The result shows no wrinkle but instead the effect of bridging. The material is in contact with the surface of the tooling only, where the grippers push the material onto it (Fig. 19). Once the lower robot is removed from the surface, the bridging effect disappeared and the cut-piece is in contact with the tooling across its entire area.

Here, the resulting deviation is the opposite compared to the first positioning strategy. Due to each gripper's individual movement along their local perpendicular on the tooling surface, the distance between the gripper's increases. The final positions of the grippers define the length $l_{\mathrm{t}}$ at the tooling surface. Bridging effects occur as soon as $l_{\mathrm{t}}$ exceeds the length $l_{\mathrm{f}}$ of the cut-piece. Figure 20 shows an extreme case of bridging, where the material spans the whole distance between the grippers.

Again, after the test, an assessment of the cut-piece showed no deterioration and it was prepared for re-use.

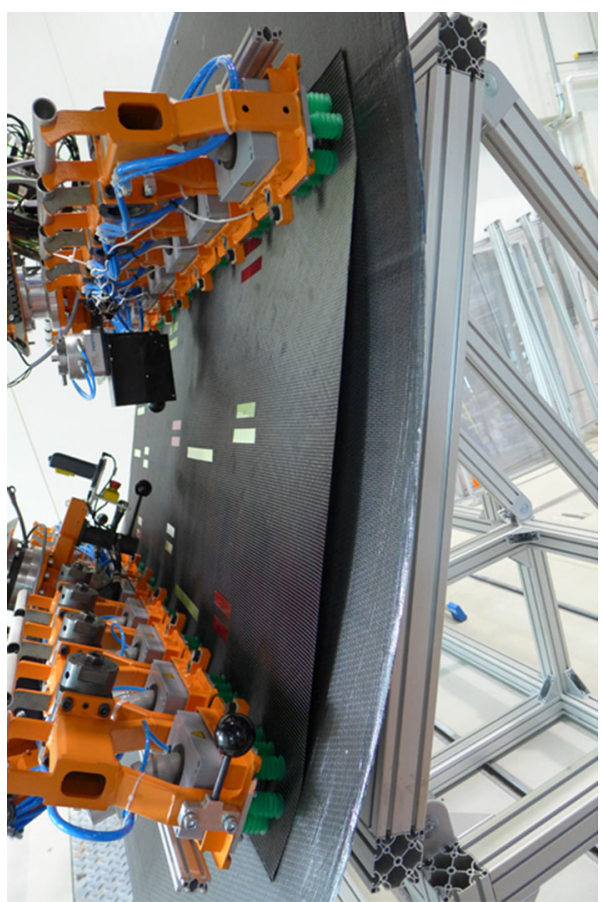

Fig. 19 Material tension after the positioning process

Fig. 20 Draping test 2

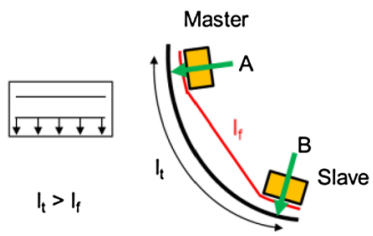

The third strategy avoids the effect of bridging in an attempt to match the lengths $l_{\mathrm{t}}$ and $l_{\mathrm{f}}$. Since the reason for bridging is the increasing distance between the two grippers during their final positioning movements, the strategy involves a correction during the pick-up process. An additional translational movement of the robots towards each other during the pick-up process is done. It is performed simultaneously with the initial rotation around the gripper's longitudinal axis and their movement upwards from the table. Due to the change of the gripper distance, the inclination of the grippers has to be adapted to maintain the catenary. The determination of the new inclination is not a part of this work.

A parameter A_1 has been introduced in the robotics program which defines the translational movement of the robots towards each other. This parameter achieves an equal change of the position of both robots by being added to the position value of the master and being subtracted from the position value of the slave. Figure 21 shows this phenomenon schematically. 


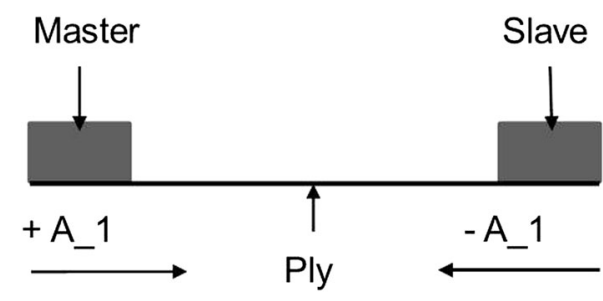

Fig. 21 A_1 parameter for master and slave

Draping test 2 was performed with A_1 $=0$. The earlier described pick-up process was generated for this value. For the next tests, the value of $A_{-} 1$ is varied $\left(A_{-} 1=1\right.$, A_1 = 2, and $A \_1=3$ ) to determine its influence for the drop-off process. The distance between both robots at the end of the pick-up process is reduced two times of the A_1 value in millimeters. The movement and positions of the master are not influenced by A_1 due to the manually taught positions. Induced by the geometrical link of the robots with increasing values of A_1, the position of the slave is getting closer to the master's one. Therefore, the drop-off distance is reduced. After each test with a different A_1 value, the lower position of the cut-piece edge is determined. As a reference, the cut-piece position for A_1 $=0$ is used.

The result is that with increasing values for $\mathrm{A}_{-} 1$, the bridging effect is reduced and the best drop-off result is achieved for $A \_1=3$. The material lies flat and smooth at the surface of the tooling and bridging disappeared completely. When the slave is removed from the surface, the cut-piece does not move. Note that the parameter A_1 depends on local curvature, cut-piece size, and thus the individual plybook of a lays up. Figure 22 shows the result of this drop-off process. An additional observation is that the value of the reduced distance of the grippers induced by A_1 is not equal to the change of the position of the lower cut-piece edge compared to the position of this edge for A_1 $=0$. Table 1 shows the applied values for the A_1 parameter, the resulting reduction of the gripper distance after pick-up, and the difference of the position of the lower cut-piece edge for the different A_1 values compared to A_ $1=0$.

It seems that the resulting differences between the lower cut-piece edge positions and the values of the reduced gripper distance are for all the performed tests round about $1 \mathrm{~mm}$. In the theory, a small difference between the change of gripper distance and the change of lower edge position would be expected due to the geometry of a circular segment. However, the uniform delta of $1 \mathrm{~mm}$ in this particular case is suspected due to a measurement resolution of only $1 \mathrm{~mm}$. To derive further conclusions, an increase of measurement resolution and further tests would be required.

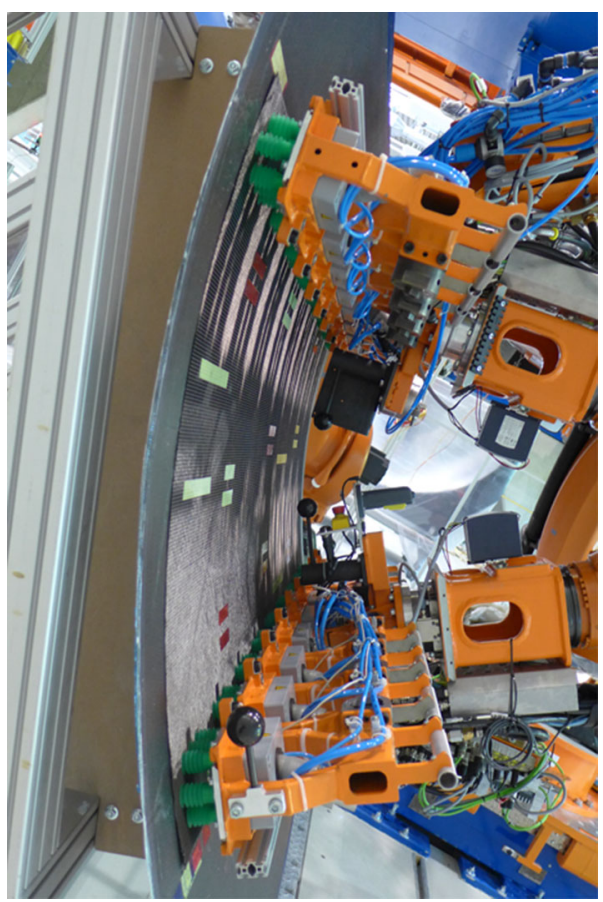

Fig. 22 Drop-off position for A_1 $=-3 / 3$

Table 1 Drop-off distances dependent on A_1

\begin{tabular}{lll}
\hline A_1 & $\begin{array}{l}\text { Reduced gripper } \\
\text { distance }(\mathrm{mm})\end{array}$ & $\begin{array}{l}\text { Difference of the lower cut-piece edge } \\
\text { positions compared to A_1 =0 (mm) }\end{array}$ \\
\hline 0 & 0 & 0 \\
1 & 2 & 3 \\
2 & 4 & 5 \\
3 & 6 & 7 \\
\hline
\end{tabular}

From the second to the third draping tests, the distance between the grippers is reduced to approximate $l_{\mathrm{t}}$ and $l_{\mathrm{f}}$. When both values are equal, bridging is avoided and the result of Fig. 23 is reached. This draping result is satisfying for the automated preforming process.

Tests showed that it is not important which robot is moving to the drop-off position at first or if it is a synchronized movement. It is only important that the movement at the drop-off position is done perpendicular to the surface.

\subsubsection{Add-on to the automated preforming process}

For the described tests, the pick-up and drop-off points of the cut-pieces were generated by a manual teaching process. This needs improvement for the automated preforming process, because it is a time consuming and non-precise process. For that reason, a function of the CAD tool CATIA is used to generate the pick-up and drop-off points of all the cut-pieces. 
Fig. 23 Draping test 3

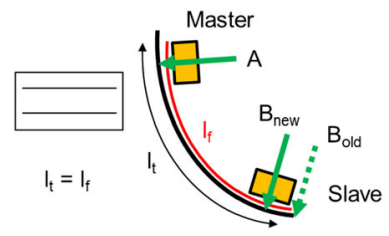

At first, the surface of the half shell tooling is scanned by a laser-tracking system. This scan is used to remodel the tooling surface in CATIA. Onto this generated surface, the cut-pieces of the plybook are placed. With the help of an XML-export, a set of points describing the two-dimensional cut-piece contour is transferred to Excel. The pickup points of the grippers at the surface of the material are generated with this two-dimensional contour of the cutpieces. The direction of the $x$ - and $y$-axes of the coordinate system is derived from the contour, too. After that the pickup points and the direction of the axes are transferred back to CATIA. To get the drop-off points in $3 \mathrm{D}$, a connection of the two-dimensional contour of the cut-pieces and the three-dimensional tooling must be established. The plybook contains information about the positions of the cutpieces on the tooling, whereas the pick-up points on the table were derived from the two-dimensional contours of the cut-pieces. With this information, the drop-off points and the direction of the $x$ - and $y$-axes can be determined by a geometry transfer in CATIA. The orientation of the $z$-axis can be calculated by the vector product. Note, the position and orientation of the coordinate systems at the pick-up and drop-off points are used to determine the position and orientation of the grippers. The directions have to be identical at both positions to have the same orientation of the grippers. The generated grip points are then transferred from CATIA into a robot language and can, therefore, be used for the preforming process [14]. An improvement of this approach is described in [15].

\section{Application of the developed automation steps}

The process details from Sect. 5 are now transferred from the process with a single cut-piece to an automated preforming process including all the cut-pieces of the half shell demonstrator. All the developed automated steps up to this point including the CAD function to generate the pick-up and drop-off points are used for this test.

\subsection{The test setup at the MFZ}

The experimental setup is built at the multifunctional robotic cell of the DLR Augsburg [16]. This cell is $32.5 \mathrm{~m}$ in length, $16 \mathrm{~m}$ of width, and $7 \mathrm{~m}$ of height. Five robots are

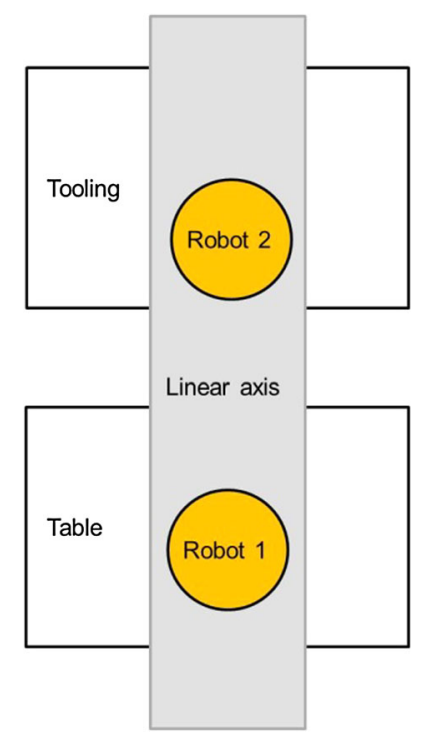

Fig. 24 Schematically test setup in top view

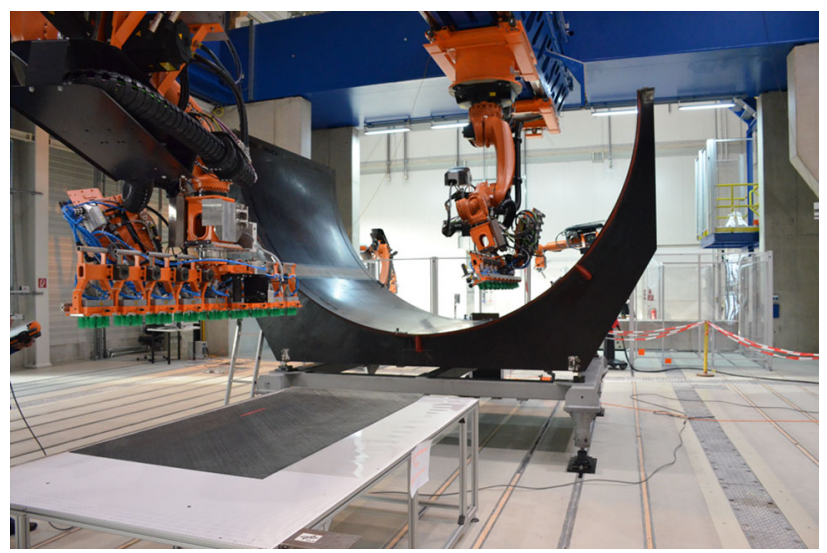

Fig. 25 Test setup at the MFZ

mounted top down on three linear axes. This configuration provides maximum accessibility for the robotics on an obstacle-free shop-floor. For the experiment, two robots at the central linear axis are used together with the software package Roboteam. The grippers are attached at the robots with an orientation of $0^{\circ}$. A table is used to provide the cutpieces, and the tooling is the half shell with a radius of $1977 \mathrm{~mm}$ and a surface length of $6204 \mathrm{~mm}$. The full set of cut-pieces in the plybook is to be picked-up, transported, positioned, and draped. Figure 24 shows the complete test setup schematically in top view and Fig. 25 the real setup.

\subsection{Production of the half shell demonstrator}

To start with the preforming process, the first cut-piece of the plybook is placed manually at the table. To generate a 
reproducible positioning at the table, a positioning system is installed at the edges of the table. Only one cut-piece can be placed at once at the table, because the vacuum suction of the bellows would lift up several cut-pieces. By reducing the vacuum suction with the proportional valve, it would not be high enough to securely pick up at least one cut-piece. The first cut-piece of each sequence which is placed at the tooling is the one in the center (Fig. 4). After that the sequence is filled in circumferential direction to the right and to the left. This process is repeated for a total of 112 cut-pieces until the lay-up is completed. An exemption was made for the first sequence. To achieve the necessary adhesion and get a smooth surface for the following cut-pieces, sequence 1 is placed by hand at the tooling. To fix these cut-pieces, an additional epoxy resin adhesive called SAERFIX is used.

All the other skin layers and frame layers are placed in an automated process using the grippers. For the drop-off process, A_1 $=3$ is used to avoid bridging and the movement from the preposition to the drop-off position is done without GEOLINK to avoid wrinkles. To fix the cut-pieces among each other, the heating elements of the grippers are pushed at the material directly after drop-off. The temperature of the heating elements is at least $102{ }^{\circ} \mathrm{C}$ which is the melting temperature of the binder and they stay at the material for $1 \mathrm{~min}$. When the material is fixed the grippers move to their home position in the program. The edges of the cut-pieces are additionally fixed with an iron. This is necessary, because the weight of the entire preform cannot be held by the small fixed points of the heating elements. The small cut-pieces at the outer edges of a sequence are placed at the tooling with just one gripper. They are so small that one gripper was found to be sufficient. The StringerBases and Stringers are handled with one gripper too. After the preforming is finished, an automated measurement of the fiber orientations of the last sequence is done.

\section{Results and discussion}

\subsection{Results}

The draping process for large textile cut-pieces has been implemented for the first time using collaborative robots in a near-industrial environment. Process steps have been transferred to a full-scale fuselage section considering the complete preforming process chain. The following results have been achieved:

- Proof of feasibility of preforming with collaborative robots.

- Development and verification of a robotic sequence for pick-up, transport, positioning, and draping of the cutpieces.
- Experimental assessment of potential use of the catenary shape for process optimization.

- Integration and experimental verification of a singlecut-piece process into a complete process chain.

- Successful preforming of a fuselage section.

- Identification of key parameters for manufacturing design.

\subsection{Discussion}

The results show that the automation of the preforming process is possible. Except of sequence 1, all the cut-pieces are placed by two collaborating robots at the half shell tooling. The generated movements for the pick-up and drop-off process are validated with the tests at the multifunctional cell. No wrinkle, bridging, or distortion of the material appeared. The automated measurement of the fiber orientations of the last sequence showed that no deformation of the fibers occurred.

A visual assessment of the drop-off quality shows that most of the cut-pieces are placed with an overlap to each other. The size of these overlaps increases at higher sequences. The reason for this could be the size of the cutpieces which is equal for all skin layers and frame layers. For the first half shell demonstrator, which was built by hand, this error did not occur, because the cut-pieces were manually placed and adjusted without a gap and overlap. The contour for placement shown at the tooling by two laser based projectors was not infinitesimal thin. Therefore, the problem was not noticed during the manual process. For the automated process, it could be seen that the size of the cut-pieces must decrease with increasing layer structure. The reason for this is the decreasing radius which is induced by the cut-pieces already lying at the tooling. This means compared to a manual lay-up, the observed overlaps result on one hand from an incorrect consideration of the lay-up thickness and on the other hand from absolute positioning rather than relative to neighboring cut-pieces. For the design of the next half shell demonstrator, it is important to change the material sizes and adapt them to the different drop-off radii. Then, the overlaps have to be checked again.

\section{Outlook}

\subsection{Further developments}

The main focus of this paper was a transfer of the manual preforming process to an automated approach. The next step is an improvement of the automated preforming steps to increase process stability and get a satisfying and 
repeatable quality of the preform. A particular focus will be on prevention of overlaps and precise edge to edge placement of the cut-pieces. Next, an assessment and increase of the layup rate will be necessary to improve process efficiency.

In addition, an automated consideration of the catenary will be integrated in more detail in an approach to link theoretical and practical work.

\subsection{Transfer of the process}

The automated preforming process steps developed in this paper in combination with the described grippers and the system of collaborating robots so far have been used for a single-curved target structure. A transfer of these process steps to different target structures is one of the next investigation steps.

In the previous work on a doubly curved pressure bulkhead [2], gripper systems were developed to pick up and drape the stiffening layers by single-robot systems. In this particular application, large cut-pieces covering the full component lengths of up to $5 \mathrm{~m}$ would be well suited for a cooperating multi-robot approach. A combination of the single-robot pick-and-place approach and the multirobot draping system from this paper might be a step towards an efficient lay-up process.

\section{Summary and conclusions}

In summary, it can be said that a basis for a fully automated preforming process has been developed. It is possible to pick up, transport, and place the cut-pieces without damage from a table to a tooling. This work is done by two collaborative robots. Each carrying a gripper as a positioning and handling system. The pick-up and drop-off points are calculated in CATIA to avoid the manually teaching process. With this, the aims 1 and 2 of automation are achieved.

From the results, the next steps for optimization are identified to improve the quality of the component. Most important is precise positioning of the cut-pieces during the drop-off process and the elimination of the overlaps between the different cut-pieces.

Acknowledgments This work is a team effort and would not have been possible without manifold contributions from our industrial partner Kuka Systems GmbH and our colleagues at the DLR. In particular, we would like to thank Dr. A. Schuster, F. Krebs, and L. Larsen for their enduring help and input. Funding was provided by the City of Augsburg and the Federal State of Bavaria.

\section{References}

1. Ehrenstein, G.W.: Faserverbund-Kunststoffe: Werkstoffe-Verarbeitung-Eigenschaften. Carl Hanser Verlag, Munich (2006)

2. Gerngross, T., Nieberl, D.: Automated manufacturing of large, three-dimensional CFRP parts from dry textiles. CEAS Aeronaut. J. 7, 214-257 (2016). doi:10.1007/s13272-016-0184-5

3. Gerngross, T., Krebs, F., Buchheim, A.: Automated production of large CFRP preforms: challenges and solutions along the process chain. Composites Manufacturing, Frankfurt (2012)

4. Schuster, A., Kuehnel, M., Kupke, M.: Automated preforming of curved thermoplastic organic sheets. SAMPE Europe Conference, Amiens (2015)

5. Gerngross, T.: Automatisierung der Produktion von Faserverbundbauteilen in der Luftfahrt. Ingenieurspiegel Luftfahrt, pp. 50-52. Public Verlag, Bingen (2014)

6. Gerngross, T., Schmidt, T., Krebs, F.: Herausforderungen für die Automation von robusten Produktionsprozessen für Faserverbundstrukturen. 1. Augsburger Produktionskolloquium, Augsburg (2011)

7. Newell, G.C., Khodabandehloo, K.: Modelling flexible sheets for automatic handling and lay-up of composite components. Advanced Manufacturing and Automation Research Centre, University of Bristol (1994)

8. Irvine, H.M.: Cable structure. Penerbit ITB, Indonesia (1988)

9. Braun, G., Buchheim, A., Fischer, F., Gerngross, T.: Handgeführter Endeffektor für die automatisierte Handhabung von textilen Zuschnitten. Deutscher Luft- und Raumfahrtkongress, Stuttgart (2013)

10. Sener, D.: Handling von Faserhalbzeugen in der automatisierten CFK-Produktion. Studienarbeit, Institut für Bauweisen und Strukturtechnologie, DLR Stuttgart, Augsburg (2011)

11. Larsen, L., Braun, G.: Entwurf und Test eines Saugsystems für das automatisierte Handling von Kohlefasergelegen. Institut für Bauweisen und Strukturtechnologie, DLR Stuttgart, Augsburg (2011)

12. Braun, G., Gerngross, T.: Entwicklung eines Endeffektors für das Handling von Kohlefasergelegen. Institut für Bauweisen und Strukturtechnologie, DLR Stuttgart, Augsburg (2011)

13. Kuka Systems GMBH: KUKA.RoboTeam 2.0. Augsburg (2013)

14. Schuster, A.: Computer Vision für die CFK-Produktion: Autonomes Greifen von individuellen Preforms. Deutscher Luft- und Raumfahrtkongress, Augsburg (2014)

15. Schuster, A.: Autonomes System zur Herstellung von Organoblechen und Preforms. Dissertation, University Augsburg, Augsburg (2015)

16. Krebs, F., Larsen, L., Braun, G., Dudenhausen, W.: Design of a Multifunctional Cell for Aerospace CFRP Production. 23rd International Conference on Flexible Automation \& Intelligent Manufacturing, Porto (2013) 